\title{
Recent results in (exotic) charmonium spectroscopy from BESIII
}

\author{
Aiqiang Guo ${ }^{1, a}$ \\ ${ }^{1}$ Institute of High Energy Physics, Chinese Academy of Sciences, Beijing, China
}

\begin{abstract}
We present the recent progress on the (exotic) charmonium spectroscopy from BESIII experiment, This paper focus on the observation of charged charmonium like states and their isospin partners from BESIII, including $Z_{c}(3900)^{ \pm, 0}, Z_{c}(4020)^{ \pm, 0}, Z_{c}(3885)^{ \pm}$and $Z_{c}(4025)^{ \pm}$. In addition, the observation of $\mathrm{X}(3872)$ from radiative decay of the $\mathrm{Y}(4260)$ and $e^{+} e^{-} \rightarrow \omega \chi_{c 0}$ are also introduced.
\end{abstract}

\section{Introduction}

The discovery of charmonium was a watershed moment in the establishment of Quantum Chromodynamics (QCD). Since that time, theoretical and experimental investigations in quarkonium physics have continued to be pillars of the international research effort in QCD.

One of the most basic properties of QCD is its spectrum. Dramatic progress can be expected in the spectroscopy of charmonium above the open-charm threshold because experiments have uncovered entirely new exotic hadronic states in this region in the past decade. The common properties of these states are relatively narrow widths and strong couplings to hidden-charm final states, therefore, it is hard to be explained that all these resonances are charmonia. Understanding the QCD spectrum in charmonium regions is one of the most interesting and exciting challenges to theorists today.

Comparing with the hadron colliders, the main strength of $e^{+} e^{-}$colliders is the capability to obtain large datasets at or near charmonium vector state masses with well-known initial-state quantum numbers and kinematics. BESIII is the only experiment currently taking data using $e^{+} e^{-}$collisions in the charmonium region. It has collected about $5 \mathrm{pb}^{-1}$ dataset above $\sqrt{s}=4 \mathrm{GeV}$ and yields fruitful results on the (exotic) charmonium spectroscopy. In this paper, we will introduce the recent progress from BESIII.

\section{Observation of charged charmonium-like states}

Over the past decade, many additional $c \bar{c}$ mesons have been observed in experiment. The list of these neutral $c \bar{c}$ mesons, which were labeled as $\mathrm{X}, \mathrm{Y}$, or $\mathrm{Z}$, has grown to more than a dozen states. Although they are candidates for exotic clusters due to their unexpected properties, such as charmonium hybrids $(c \bar{c} g)$ or charmonium

\footnotetext{
ae-mail: guoaq@ihep.ac.cn
}

tetraquarks $(c \bar{c} q \bar{q})$, none of these neutral $c \bar{c}$ mesons is manifestly exotic. Since their $J^{P C}$ or flavor quantum numbers are compatible with conventional charmonium, therefore, we could not exclude these states from simply being complicated manifestations of the strong interactions between hadrons.

This situation has been changed by the recent discoveries of charged quarkonium-like mesons in the $b \bar{b}$ and $c \bar{c}$ sectors. In November 2011, the Belle Collaboration discovered the $Z_{b}^{+}(10610)$ and $Z_{b}^{+}(10650)$ [1], whose decays into $\Upsilon \pi$ reveal their constituents to be $b \bar{b} u \bar{d}$. In April 2013, the BESIII Collaboration discovered the $Z_{c}^{+}$(3900) [2], whose decays into $J / \psi \pi^{+}$reveal its constituents to be $c \bar{c} u \bar{d}$. This state was almost immediately confirmed by the Belle Collaboration [3]. These charged tetraquark mesons are relatively narrow although they are above the thresholds for decays into pairs of heavy-light mesons. The discoveries of these manifestly exotic states are a glimpse into a sector of the QCD spectrum that has remained hidden for all these decades.

Recently, a number of charged charmonium-like states and their isospin partners have been observed by the BESIII collaboration. These results provide important informations for understanding the internal structure of these exotic states. In the following sections, we will introduce the observations of $Z_{c}(3900)$ and its partner states from BESIII.

\subsection{Observation of $Z_{c}(3900)$ and $Z_{c}(3885)$}

The discovery of $Z_{b}^{+}(10610)$ and $Z_{b}^{+}(10650)$ indicates the possible existence of charged bottomonium-like states, which must have at least four constituent quarks to have a non-zero electric charge, rather than the two in a conventional meson. By analogy, this suggests there may exist interesting substructure in the $Y(4260) \rightarrow \pi^{+} \pi^{-} J / \psi$ process in the charmonium region.

A study of the process $e^{+} e^{-} \rightarrow \pi^{+} \pi^{-} J / \psi$ at a centerof-mass (CM) energy of $\sqrt{s}=4.260 \mathrm{GeV}$ is performed with a $525 \mathrm{pb}^{-1}$ data sample collected with the BESIII 


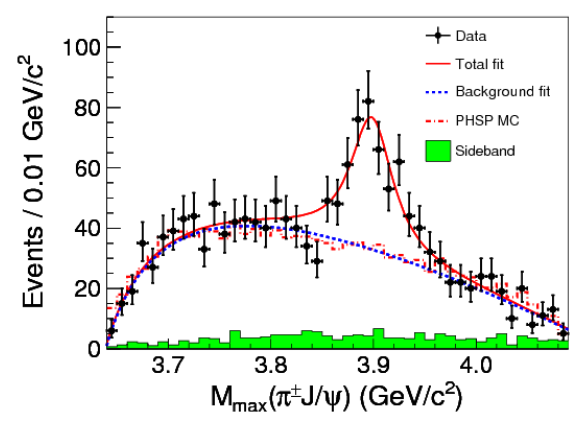

Figure 1. Fit to the $M_{\max }\left(\pi^{ \pm} J / \psi\right)$ distribution as described in the text. Dots with error bars are data; the red solid curve shows the total fit, and the blue dotted curve the background from the fit; the red dot dashed histogram shows the result of a phase space MC simulation; and the green shaded histogram shows the normalized $J / \psi$ sideband events.

detector [2]. A charged structure in the $\pi^{ \pm} J / \psi$ invariant mass spectrum was observed referred to as $Z_{c}(3900)$. An unbinned maximum likelihood fit to the distribution of $M_{\max }\left(\pi^{ \pm} J / \psi\right)$, which was shown in Fig. 1 , determined the mass and width of $Z_{c}(3900)$ to be $(3899.0 \pm 3.6 \pm 4.9)$ $\mathrm{MeV} / \mathrm{c}^{2}$ and $(46 \pm 10 \pm 20) \mathrm{MeV}$, respectively. In addition, the production ratio was measured to be $R=$ $\frac{\left(e^{+} e^{-} \rightarrow \pi^{ \pm} Z_{c}^{\mp} \rightarrow \pi^{+} \pi^{-} J / \psi\right)}{\left(e^{+} e^{-} \rightarrow \pi^{+} \pi^{-} J / \psi\right)}=(21.5 \pm 3.3 \pm 7.5) \%$.

Inspired by the discovery of charged $Z_{c}(3900)$, its isospin partner $Z_{c}(3900)^{0}$ was also observed in events of $e^{+} e^{-} \rightarrow \pi^{0} \pi^{0} \mathrm{~J} / \psi$ with an integrated luminosity of approximately $2.8 \mathrm{fb}^{-1}$ datasets from $\sqrt{s}=4.19 \mathrm{GeV}$ to 4.42 $\mathrm{GeV}$ [4]. An unbinned maximum likelihood fit to the distribution of $M_{\max }\left(\pi^{0} \mathrm{~J} / \psi\right)$, as shown in Fig. 2, determined the mass and width of $Z_{c}(3900)^{0}$ to be $(3894.8 \pm 2.3 \pm 2.6)$ $\mathrm{MeV} / \mathrm{c}^{2}$ and $(29.6 \pm 8.2 \pm 7.3) \mathrm{MeV}$, respectively. The discovery of $Z_{c}(3900)^{0}$ indicates the isospin triplet state $Z_{c}(3900)$ has been established.

Since the mass of $Z_{c}(3900)$ is close to the $D \bar{D}^{*}$ mass threshold, One natural explanation is that the $Z_{c}$ (3900) state is an S-wave $D \bar{D}^{*}$ molecular state or molecular-type resonance. The BESIII experiment performed a study of the process $e^{+} e^{-} \rightarrow \pi^{ \pm}\left(D \bar{D}^{*}\right)^{\mp}$ using the same data sample at $4.26 \mathrm{GeV}$ [5]. In this analysis, to increase the detection efficiency, only the bachelor $\pi^{ \pm}$and one D meson were detected, the $\bar{D}^{*}$ was inferred from energy-momentum conservation.

In the recoil mass of the bachelor $\pi^{ \pm}$, an enhancement was observed near the $D \bar{D}^{*}$ mass threshold, named $Z_{c}(3885)$. Fits to the mass spectrum with a massdependent-width BW function, as shown in Fig. 3, the pole mass and width were determined to be $\mathrm{M}=(3885.9 \pm 1.5 \pm$ 4.2) $\mathrm{MeV} / \mathrm{c}^{2}$ and $\Gamma=(24.8 \pm 3.3 \pm 11.0) \mathrm{MeV}$. Assuming the $Z_{c}(3885)$ and the $Z_{c}(3900)$ are the same state, the ratio of partial decay widths is determined to be $\frac{\left(Z_{c}(3885) \rightarrow D \bar{D}\right)}{\left(Z_{c}(3900) \rightarrow \pi J / \psi\right)}=$ $6.2 \pm 1.1 \pm 2.7 \%$. This ratio is much smaller than typical values for decays of conventional charmonium state above the open charm threshold.

In addition, the BESIII experiment also studied the quantum numbers of $Z_{c}(3885)$ by investigating the polar
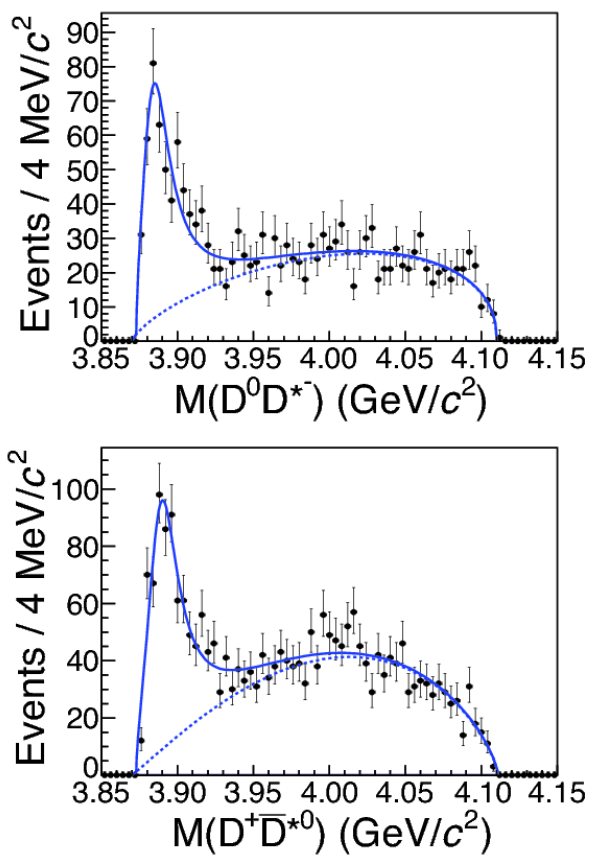

Figure 3. The $M\left(D^{0} \bar{D}^{*-}\right)$ (top) $M\left(D^{+} \bar{D}^{* 0}\right)$ (middle) distributions for selected events.

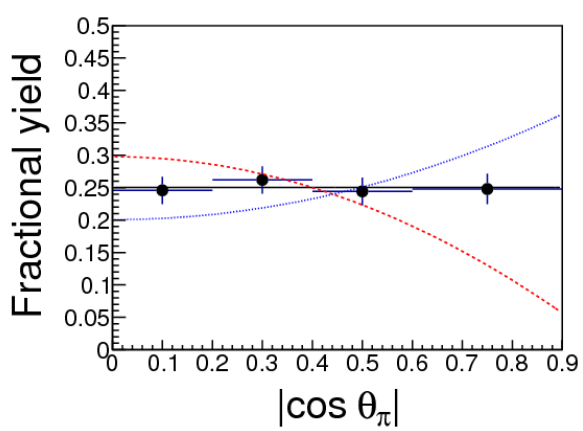

Figure 4. $1 / N_{t o t} d N / d|\cos \theta|$ as a function of $\left|\cos \theta_{\pi}\right|$ for $\mathrm{Zc}(3885)$ events in data. The solid, dashed and dotted curves show expectations for $J^{P}=1^{+}, 0^{-}$, and $1^{-}$, respectively.

angle distribution of the bachelor pion. The data support the expectation for $J^{P}=1^{+}$as shown in Fig. 4, which is the efficiency corrected fractional signal yield as a function of $\left|\cos \theta_{\pi}\right|$.

\subsection{Observation of $Z_{c}(4020)$ and $Z_{c}(4025)$}

After the observation of $Z_{c}$ (3900), one can easily infer that there may be new similar structures existing in the mass of one pion and a higher mass charmonia, such as $h_{c}, \psi(2 S)$ etc. The CLEOc has observed a large cross section of $e^{+} e^{-} \rightarrow \pi^{+} \pi^{-} h_{c}$ in the $\sqrt{s}$ above open charm thresholds. Therefore, a study of $e^{+} e^{-} \rightarrow \pi^{+} \pi^{-} h_{c}$ is expected to search for new charged charmonium-like states.

BESIII studied the process of $e^{+} e^{-} \rightarrow \pi^{+} \pi^{-} h_{c}$ using data samples taken at $\sqrt{s}$ from 3.90 to $4.42 \mathrm{GeV}$ [6]. The $h_{c}$ was reconstructed through its electric-dipole(E1) 

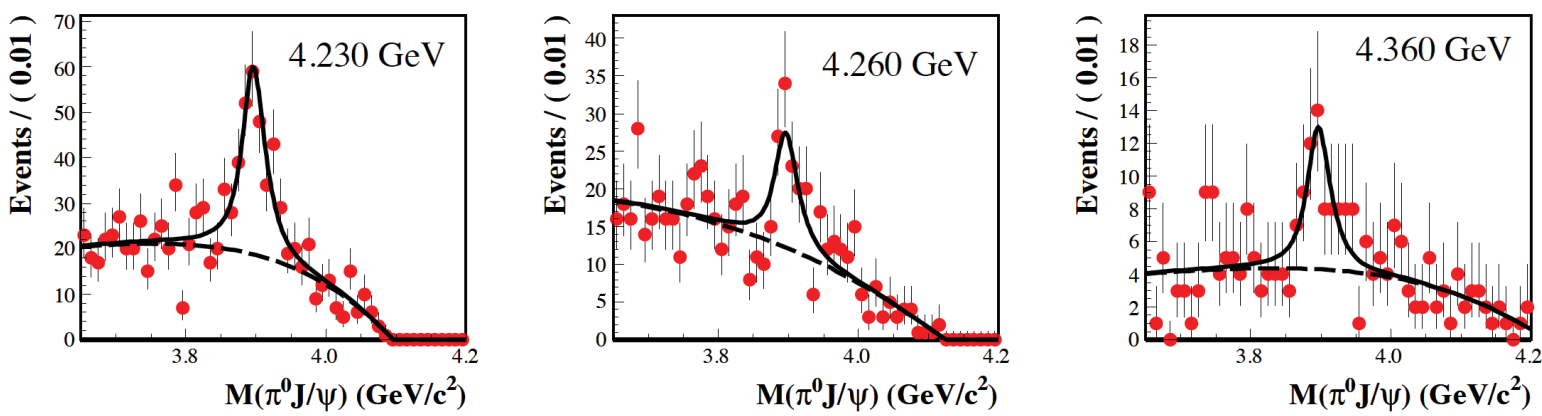

Figure 2. The simultaneously fitted $\pi^{0} J / \psi$ mass spectra for $\mathrm{Ecm}=4.230 \mathrm{GeV}, 4.260 \mathrm{GeV}$ and $4.360 \mathrm{GeV}$ in data. (BESIII preliminary results)

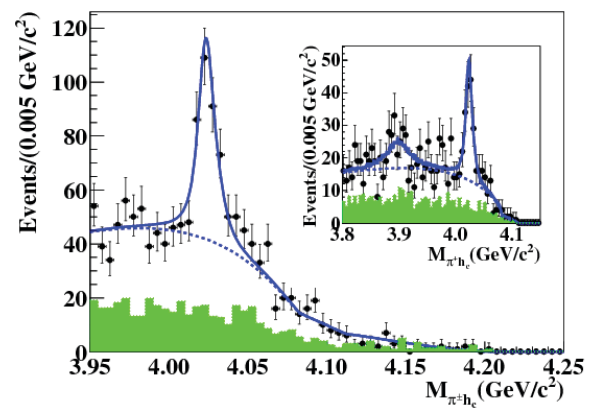

Figure 5. Sum of the simultaneous fit to the $M\left(\pi^{ \pm} h_{c}\right)$ at 4.23 , 4.26 , and $4.36 \mathrm{GeV}$; the inset shows the sum of the simultaneous fit to the $M\left(\pi^{+} h_{c}\right)$ distributions at 4.23 and $4.26 \mathrm{GeV}$ with $Z_{c}(3900)$ and $Z_{c}(4020)$. Dots with error bars are data; shaded histograms are normalized $h_{c}$ sideband background; the solid curves show the total fit, and the dotted curves the backgrounds from the fit.

transition $h_{c} \rightarrow \gamma \eta_{c}$, while $\eta_{c}$ was reconstructed using 16 hadronic decay modes: $p \bar{p}, 2\left(\pi^{+} \pi^{-}\right), 2\left(K^{+} K^{-}\right)$, $K^{+} K^{-} \pi^{+} \pi^{-}, p \bar{p} \pi^{+} \pi^{-}, 3\left(\pi^{+} \pi^{-}\right), K^{+} K^{-} 2\left(\pi^{+} \pi^{-}\right), K^{+} K^{-} \pi^{0}$, $p \bar{p} \pi^{0}, K_{S}^{0} K^{ \pm} \pi^{\mp}, K_{S}^{0} K^{ \pm} \pi^{\mp} \pi^{ \pm} \pi^{\mp}, \pi^{+} \pi^{-} \eta, K^{+} K^{-} \eta, 2\left(\pi^{+} \pi^{-}\right) \eta$, $\pi^{+} \pi^{-} \pi^{0} \pi^{0}$ and $2\left(\pi^{+} \pi^{-}\right) \pi^{0} \pi^{0}$.

From the analysis of Dalitz plot of $\pi^{+} \pi^{-} h_{c}$ system at $4.23,4.26$, and $4.36 \mathrm{GeV}$. A structure around $4.02 \mathrm{GeV}$ was observed in the $\pi^{ \pm} h_{c}$ invariant mass spectrum, named as $Z_{c}(4020)$. A simultaneous fit to the $\pi^{ \pm} h_{c}$ mass spectrum in the three energy points with the same signal function, which were shown in Fig. 5, yields a mass of (4022.9 \pm $0.8 \pm 2.7) \mathrm{MeV} / \mathrm{c}^{2}$ and a width of $(7.9 \pm 2.7 \pm 2.6) \mathrm{MeV}$ with the statistical significance larger than $8.9 \sigma$.

To search for the potential decay of $Z_{c}(3900) \rightarrow \pi^{ \pm} h_{c}$, a $Z_{c}(3900)$ resonance with mass and width fixed to the BESIII measurement [2] was added in the fit. But the statistical significance was just about $2.1 \sigma$. The upper limits at $90 \%$ C.L. on the production cross section of $e^{+} e^{-} \rightarrow \pi^{ \pm} Z_{c}(3900)^{\mp} \rightarrow \pi^{+} \pi^{-} h_{c}$ were determined to be $13 \mathrm{pb}$ and $11 \mathrm{pb}$ at $4.23 \mathrm{GeV}$ and $4.26 \mathrm{GeV}$, respectively.

In the isospin partner process, $e^{+} e^{-} \rightarrow \pi^{0} \pi^{0} h_{c}$, with $h_{c}$ reconstructed by the same final states as in $e^{+} e^{-} \rightarrow$ $\pi^{+} \pi^{-} h_{c}$ process, the neutral partner of $Z_{c}(4020)^{ \pm}$was investigated using the same data samples at 4.23, 4.26 and

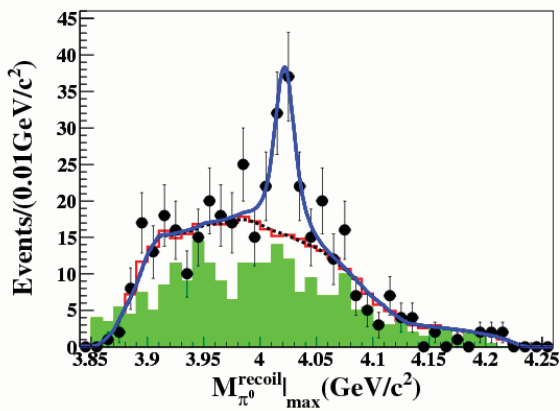

Figure 6. Sum of the simultaneous fit to the $M_{\max }\left(\pi^{0} h_{c}\right)$ distributions at 4.230, 4.260, and $4.36 \mathrm{GeV}$. Dots with errors bars are data; the green shaded histogram shows the normalized $h_{c}$ sideband events; the black dashed curve is the background from the fit; the red histogram shows the result from a phase space MC simulation. The solid blue line shows the total fit.

$4.36 \mathrm{GeV}$ [7]. In the $\pi^{0} h_{c}$ mass spectrum, an obvious peak around $4.02 \mathrm{GeV}$ was observed, as shown in Fig. 6. Applying a simultaneous fit to the $M_{m a c}\left(\pi^{0} h_{c}\right)$ mass spectrum in the three energy points with width fixed to the value of charged channel, the mass of neutral $Z_{c}(4020)$ was determined to be $4023.6 \pm 2.2 \pm 3.9 \mathrm{MeV} / \mathrm{c}^{2}$, which is consistent with the charged $Z_{c}(4020)$. The statistical significance of the neutral $Z_{c}(4020)$ is larger than $5 \sigma$.

Since the $Z_{c}(4020)$ located in the production threshold of $D^{*} \bar{D}^{*}$, BESIII experiment also performed a study of $e^{+} e^{-} \rightarrow \pi^{ \pm}\left(D^{*} \bar{D}^{*}\right)^{\mp}$ process using $827 \mathrm{pb}^{-1}$ data at 4.26 $\mathrm{GeV}$ [8]. Similarly, only the charged D meson and the bachelor $\pi^{ \pm}$were reconstructed in the analysis. To further suppress background, one additional $\pi^{0}$ was required in the final states for the candidate events.

In the recoil mass spectrum of the bachelor $\pi^{ \pm}$, a structure near the $\left(D^{*} \bar{D}^{*}\right)$ threshold was observed (named as $\left.Z_{c}(4025)\right)$ as shown in Fig. 7. A fit to the mass spectrum using unbinned maximum likelihood method yields a mass of $(4026.3 \pm 2.6 \pm 3.7) \mathrm{MeV} / \mathrm{c}^{2}$, and a width of $(24.8 \pm 5.6 \pm 7.7) \mathrm{MeV}$. The Born cross section of $e^{+} e^{-} \rightarrow \pi^{ \pm}\left(D^{*} \bar{D}^{*}\right)^{\mp}$ was calculated to be $(139 \pm 9 \pm 15)$ pb. Assuming the $Z_{c}(4020)$ and the $Z_{c}(4025)$ are the same state, the ratio of partial decay widths is determined to be 


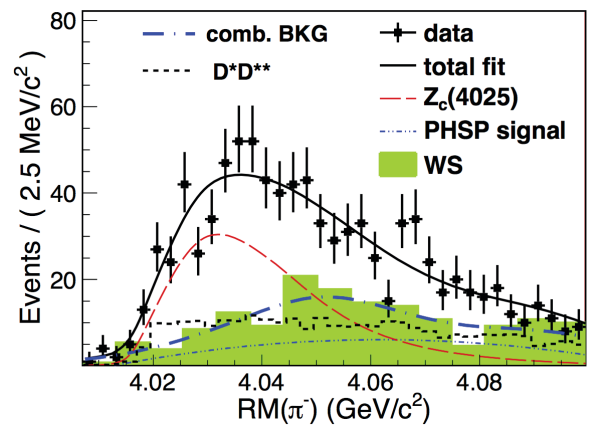

Figure 7. Unbinned maximum likelihood fit to the $\pi^{-}$recoil mass spectrum from $e^{+} e^{-} \rightarrow \pi^{ \pm}\left(D^{*} \bar{D}^{*}\right)^{\mp}$ process.

Table 1. Summary of the charged charmonium-like states

\begin{tabular}{lcc}
\hline States & Mass $\left(\mathrm{MeV} / c^{2}\right)$ & Width $(\mathrm{MeV})$ \\
\hline$Z_{c}(3900)^{ \pm}$ & $3899.0 \pm 3.6 \pm 4.9$ & $46 \pm 10 \pm 20$ \\
$Z_{c}(3900)^{0}$ & $3894.8 \pm 2.3 \pm 2.6$ & $29.6 \pm 8.2 \pm 7.3$ \\
$Z_{c}(3885)^{ \pm}$ & $3885.9 \pm 1.5 \pm 4.2$ & $24.8 \pm 3.3 \pm 11.0$ \\
$Z_{c}(4020)^{ \pm}$ & $4022.9 \pm 0.8 \pm 2.7$ & $7.9 \pm 2.7 \pm 2.6$ \\
$Z_{c}(4020)^{0}$ & $4023.6 \pm 2.2 \pm 3.9$ & fixed \\
$Z_{c}(4025)^{ \pm}$ & $4026.3 \pm 2.6 \pm 3.7$ & $24.8 \pm 5.6 \pm 7.7$ \\
\hline
\end{tabular}

$\frac{Z_{c}(4025) \rightarrow D^{*} \bar{D}^{*}}{Z_{c}(4020) \rightarrow \pi^{ \pm} h_{c}}=12 \pm 5$, which is also much smaller than the conventional charmonium state above open charm threshold.

\subsection{Summary of the charged charmonium-like states}

Table 1 summarized the charged charmonium-like states covered in this paper, These states all contain at least four quarks and are close to the open-charm threshold. One natural question is what's the internal structure of these four-quark charmonium-like resonance? There are several models to explain their nature. Hadronic molecules, in which heavy-light quark-antiquark paris form heavy mesons and the mesons interact with each other through exchange of light quarks and gluons. Hadroquarkonium, in which the heavy quark pair forms a highly bound system. Tetraquarks, in which the $Q q$ and $\bar{Q} \bar{q}$ pairs form relatively tightly bound diquark and antiqiquark. To pin down the properties of these states, more measurements are needed, e.g., establishing their spin and parity, searching for more decay patterns on both open/hidden charm channels. Furthermore, similar analyses in the bottomonium system may help to solve the puzzle.

\section{Observation of $\mathrm{X}(3872)$ in}

$$
e^{+} e^{-} \rightarrow \gamma X(3872)
$$

A decade ago, a new state, the X(3872), was observed at Belle [9] and quickly confirmed by CDF [10], D0 [11], and BABAR [12]. It was hard to identify it as a missing state of the conventional charmonium spectrum as more of its properties were measured. The $\mathrm{X}(3872)$ mass is very

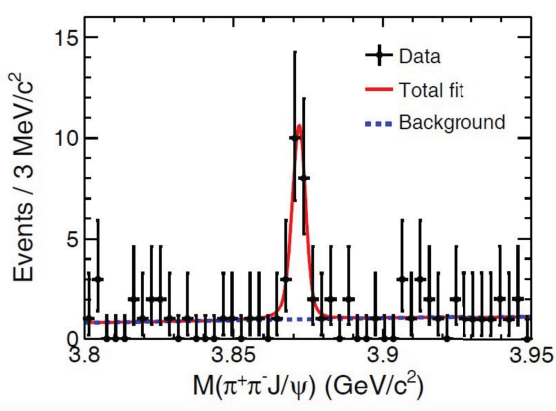

Figure 8. Fit to the summed $\pi^{+} \pi^{-} J / \psi$ invariant mass spectrum. Dots with error bars are data, and the curves are the best fit.

close to a strong decay threshold $D \bar{D}^{*}$ and its decays exhibit significant isospin violation. Both BaBar and Belle experiments observed $X(3872) \rightarrow \gamma J / \psi$, which suggests it being a C-even state $[13,14]$. The CDF and LHCb experiments determined the spin-parity of the $\mathrm{X}(3872)$ to be $J^{P}=1^{+}$by performing the angular analysis $[15,16]$. Currently, X(3872) was only observed in B meson decays or hadron collisions. Since its quantum numbers are $1^{++}$, it could also be produced through radiative transition from the vector charmonium or charmonium-like states.

The process $e^{+} e^{-} \rightarrow \gamma \pi^{+} \pi^{-} J / \psi$ has been studied in the BESIII experiment with the data samples collected at the CM energy $\sqrt{s}=4.009,4.230,4.260$, and 4.360 $\mathrm{GeV}$ [17]. Figure 8 shows the $\pi^{+} \pi^{-} J / \psi$ invariant mass spectrum summed over all the energy points and the best fit results. Obvious X(3872) signals were observed with a statistical significance of $6.3 \sigma$, the mass of the $\mathrm{X}(3872)$ was measured to be $(3872.1 \pm 0.8 \pm 0.3) \mathrm{MeV} / \mathrm{c}^{2}$.

The product of the Born cross section and the branching fraction of $X(3872) \rightarrow \pi^{+} \pi^{-} J / \psi$ as a function of CM energy are shown in Fig. 9. Since the $\mathrm{X}(3872)$ signals were not significant at $\sqrt{s}=4.009$ and $4.360 \mathrm{GeV}$, the upper limits at $90 \%$ confidence level (C.L.) were determined. The measured cross section suggests that the $\mathrm{X}(3872)$ comes from the radiative transition of $\mathrm{Y}(4260)$. If we assume the branching fraction $B\left(X(3872) \rightarrow \pi^{+} \pi^{-} J / \psi\right)=$ $5 \%$ [18] and take the cross section of $e^{+} e^{-} \rightarrow \pi^{+} \pi^{-} J / \psi$ measured by BESIII [17] into account, the fraction $R=$ $\frac{\sigma^{B}\left(e^{+} e^{-} \rightarrow \gamma X(3872)\right)}{\sigma^{B}\left(e^{+} e^{-} \rightarrow \pi^{+} \pi^{-} J / \psi\right)}$ is determined to be $11 \%$. The measured relatively large decay width near $4.260 \mathrm{GeV}$ is close to model-dependent calculations in Ref. [19].

\section{Observation of $e^{+} e^{-} \rightarrow \omega \chi_{c 0}$ at $\sqrt{s}>4$ $\mathrm{GeV}$}

The charmonium-like state $\mathrm{Y}(4260)$ was first observed in its decay to $\pi^{+} \pi^{-} J / \psi$. Although there are many theoretical models to explain its unexpected properties, its substructure is still unknown, therefore, searches for new decay modes may provide information that is useful for understanding the nature of the $\mathrm{Y}(4260)$. The production of $e^{+} e^{-} \rightarrow \omega \chi_{c J}(J=0,1,2)$ was studied in the BESIII experiment [20] based on data samples collected at 9 CM energy points from $\sqrt{s}=4.21$ to $4.42 \mathrm{GeV} . e^{+} e^{-} \rightarrow \omega \chi_{c 0}$ was ob- 


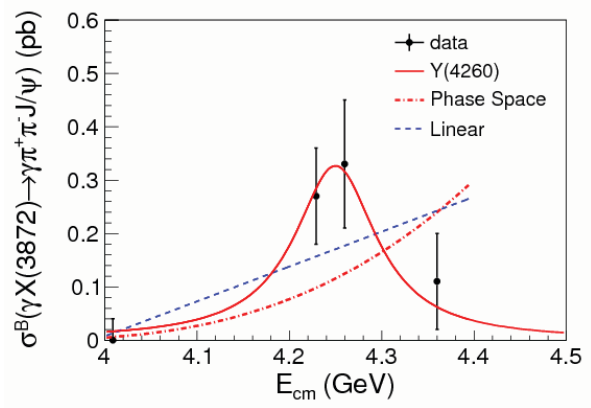

Figure 9. The fit to $\sigma^{B}\left[e^{+} e^{-} \rightarrow \gamma X(3872)\right] \times B[X(3872) \rightarrow$ $\left.\pi^{+} \pi^{-} J / \psi\right]$ with a $\mathrm{Y}(4260)$ resonance (red solid curve), a linear continuum (blue dashed curve), or a E1-transition phase space term (red dotted-dashed curve). Dots with error bars are data.
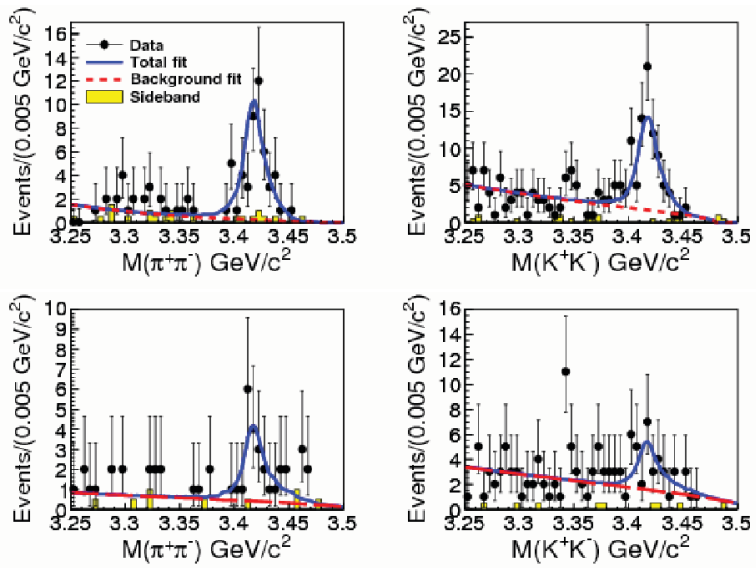

Figure 10. Fit to the invariant mass distributions $M\left(\pi^{+} \pi^{-}\right)$(left) and $M\left(K^{+} K^{-}\right)$(right). Points with error bars are data, the solid curves are the fit results, the dashed lines indicate the background and the shaded histograms show the normalized $\omega$ sideband events. The top two plots represent data at $\sqrt{s}=4.23 \mathrm{GeV}$, and the bottom two at $\sqrt{s}=4.26 \mathrm{GeV}$.

served for the first time at 4.23 and $4.26 \mathrm{GeV}$ as shown in Fig. 10, while signals of $e^{+} e^{-} \rightarrow \omega \chi_{c 1}$ and $\chi_{c 2}$ were not significant, thus the upper limits on the cross sections were determined. In this analysis, $\omega$ was reconstructed using its decay into the $\pi^{+} \pi^{-} \pi^{0}$ final state, $\chi_{c 0}$ was reconstructed via its $\pi^{+} \pi^{-}$and $K^{+} K^{-}$decays and $\chi_{c 1,2}$ was reconstructed via its decay into $\gamma \mathrm{J} / \psi$. Figure 11 shows the cross section of $e^{+} e^{-} \rightarrow \omega \chi_{c 0}$ as a function of CM energy, in which the cross section peaks at $4.23 \mathrm{GeV}$ indicating that the production of $\omega \chi_{c 0}$ does not arise from Y(4260).

\section{Summary}

Dramatic progress has been made in the charmonium spectroscopy above open charm threshold in the BESIII experiment. A new class of charged charmonium-like states has been discovered, which must contain at least 2 quark/anti-quark pairs. They provide strong evidence that more types of bound states than just mesons and baryons exist. In addition, radiative and hadronic transi-

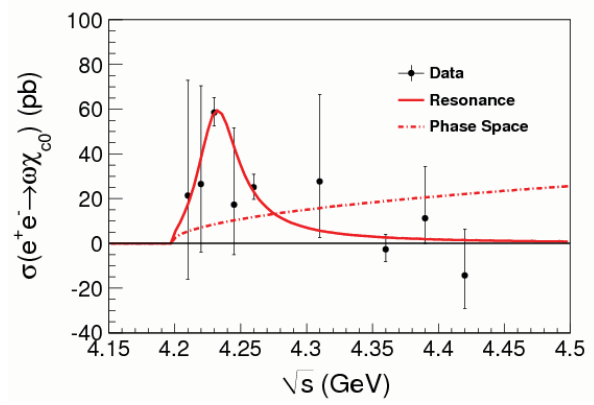

Figure 11. Fit to $\sigma\left(e^{+} e^{-} \rightarrow \omega \chi_{c 0}\right)$ with a resonance (solid curve), or a phase space term (dot-dashed curve). Dots with error bars are the dressed cross sections.

tions between higher vector charmonium(-like) states to exotic/conventional charmonium states have also been investigated. Most of XYZ states are not understood well so far, more experimental measurements and theory calculations are needed to solve the puzzle.

\section{References}

[1] A. Bondar et al. [Belle Collaboration], Phys. Rev. Lett. 108, 122001 (2012).

[2] M. Ablikim et al. [BESIII Collaboration], Phys. Rev. Lett. 110, 252001 (2013).

[3] Z. Q. Liu et al. [Belle Collaboration], Phys. Rev. Lett. 110, 252002 (2013).

[4] H. P. Peng for BESIII Collaboration, talk at the ICHEP2014, Jul 2014, Valencia, Spain.

[5] M. Ablikim et al. [BESIII Collaboration], Phys. Rev. Lett. 112, 022001 (2014).

[6] M. Ablikim et al. [BESIII Collaboration], Phys. Rev. Lett. 111, 242001 (2013).

[7] M. Ablikim et al. [BESIII Collaboration], Phys. Rev. Lett. 113, 212002 (2014).

[8] M. Ablikim et al. [BESIII Collaboration], Phys. Rev. Lett. 112, 132001 (2014).

[9] S. K. Choi et al. [Belle Collaboration], Phys. Rev. Lett. 91, 262001 (2003) [hep-ex/0309032].

[10] D. Acosta et al. [CDF Collaboration], Phys. Rev. Lett. 93, 072001 (2004) [hep-ex/0312021].

[11] V. M. Abazov et al. [D0 Collaboration], Phys. Rev. Lett. 93, 162002 (2004) [hep-ex/0405004].

[12] B. Aubert et al. [BaBar Collaboration], Phys. Rev. D 77, 111101 (2008) [arXiv:0803.2838 [hep-ex]].

[13] B. Aubert et al. [BaBar Collaboration], Phys. Rev. D 74, 071101 (2006) [hep-ex/0607050].

[14] V. Bhardwaj et al. [Belle Collaboration], Phys. Rev. Lett. 107, 091803 (2011) [arXiv:1105.0177 [hep-ex]].

[15] A. Abulencia et al. [CDF Collaboration], Phys. Rev. Lett. 98, 132002 (2007) [hep-ex/0612053].

[16] R. Aaij et al. [LHCb Collaboration], Eur. Phys. J. C 72, 1972 (2012).

[17] M. Ablikim et al. [BESIII Collaboration], Phys. Rev. Lett. 112, 092001 (2014). 
[18] C. Z. Yuan for the BESIII and Belle Collaboration, arVix:0910.3138

[19] F. K. Guo, C. Hanhart, U. G. Meissner, Q. Wang and Q. Zhao, Phys. Lett. B 725, 127 (2013).
[20] M. Ablikim et al. [BESIII Collaboration], [arXiv:1410.6538 [hep-ex]]. 